\title{
Effect of p21-activated kinase 1 inhibition on proliferation, migration and invasion of glioma
}

\section{Qinghao Yi}

Xuzhou Medical University

Tianze Chen

Xuzhou Medical University

Kunlin Zhou

Xuzhou Medical University

Qiang Ma

Xuzhou Medical University

Zhiyuan Sun

Xuzhou Medical University

Lei Wang

Xuzhou Medical University

Renhao Wang

Xuzhou Medical University

Hengliang Shi ( $\square$ shl@xzhmu.edu.cn )

Xuzhou Medical University https://orcid.org/0000-0002-9649-1910

\section{Primary research}

Keywords: Glioma, PAK1, proliferation, migration, invasion

Posted Date: March 10th, 2020

DOI: https://doi.org/10.21203/rs.3.rs-16627/v1

License: (c) (1) This work is licensed under a Creative Commons Attribution 4.0 International License.

Read Full License 


\section{Abstract}

Background: Abnormally expressed the p21-activated kinases (PAKs) are implicated in the development and treatment of glioma. Previous study has reported that PAK1 is expressed in glioma. However, the role and mechanism of PAK1 in glioma progression remain unclear.

Methods: Western blotting was employed to detect the expression of PAK1 in human glioma tissues. CCK-8, EdU and colony formation assay were applied to evaluate the effect of PAK1 inhibition on cell proliferation of glioma. The flow cytometry was utilized to examine the cell cycle distribution and apoptotic rate of glioma. Wound healing and transwell assay were exploited to investigate the effect of PAK1 inhibition on cell migration and invasion of glioma. The orthotopic xenograft glioma model was established to probe the effect of PAK1 silencing on tumor formation of U87 cell.

Results: It was showed that PAK1 was significantly upregulated in glioma tissues. Besides, high level of PAK1 was found to be associated with poor prognosis in glioma patients in TCGA database. PAK1 inhibition restrained cell proliferation, arrested cells at G1 phase, and induced cell apoptosis of glioma. PAK1 inhibition suppressed glioma cell migration and invasion. Moreover, knockdown of PAK1 dramatically decreased the protein expressions including MDM2, p38, p-p38, CyclinD1, CDK4, Bcl-2, MMP2, MMP9, Cofilin, while increased the protein levels of p53, Bax, p21 and Cleaved Caspase-3. Finally, orthotopic xenograft glioma model confirmed that silencing of PAK1 repressed the tumor formation of U87 cell transplantation.

Conclusion: Our study described that PAK1 inhibition impedes proliferation, migration and invasion of glioma cells and thus probably as a novel target for glioma therapy.

\section{Background}

Glioma is the most common primary brain tumor of human beings, histologically, it is known to originate from glial cell, according to the 2016 World Health Organization (WHO) classification, which comprises grades I-IV[1, 2]. Specially, the malignant brain tumor glioblastoma multiforme (GBM), as the grade IV glioma form, with high mortality rate, it has the worst prognosis among all glioma patients $[3,4]$. Though the current standard of treatment for newly diagnosed GBM composed of maximum surgical resection, radiotherapy and adjuvant chemotherapy with temozolomide (TMZ)[5]. However, due to its high infiltration, rapid progression and intractable resistant, the outcomes for GBM patients remain quite poor, with a median survival of less than 15 months[6]. Considerable progress has been made, whereas the overall survival (OS) rate of patients with GBM has not been significantly improved yet. Therefore, a better comprehension of the underlying molecular mechanisms of GBM, and seeking an effective targeting therapeutic strategy is necessary.

The p21-activated kinases (PAKs) are evolutionary conserved from protozoa to mammals[7]. Which belong to a family of serine/threonine protein kinases, and originally identified as downstream effectors of cell division control protein 42 (CDC42) and small GTPases RAC. The regulatory domain of PAK1 
consists of p21-binding domain (PBD) and an overlapping auto-inhibitory domain (AID). Based on their structural differences and sequence homologues, the PAKs are classified into two groups, Group I PAKs, consisting of PAKs $1-3$, and Group II PAKs, consisting of PAKs 4-6[8,9]. They are positioned at an intersection of multiple signaling pathways implicated in oncogenesis. As a member of PAKs, PAK1 is an intracellular plasma protein, which is located on chromosome 11q13 and expresses in most tissues, especially in neuronal cells of the central nervous system (CNS), thus, PAK1 has various phosphorylation substrates and interacting proteins, by means of its catalytic and scaffolding activities, performs a critical role in involving in regulation of transmitting a variety of signals controlling cytoskeleton dynamics, adhesion and fundamental cellular activities including proliferation and apoptosis[10-12]. Recently, a growing body of studies have suggested that PAK1 overexpresses in multiple malignancies, such as breast cancer, ovary cancer and colon cancer, in addition, its high expression level is positively correlated with poor prognosis of patients[10]. Briefly, these findings indicate that PAK1 plays a significant influence on tumor development and progress. However, its characters in glioma remain unknown. Therefore, it is necessary to have an in-depth exploration of whether and how PAK1 inhibition influents on cell proliferation, migration and invasion in glioma cells.

In the present study, we first demonstrated that PAK1 overexpressed in glioma and predicted poor prognosis in patients. Furthermore, we also revealed that inhibition of PAK1 by small molecule inhibitor IPA-3 and PAK1 shRNA potentially suppressed growth, proliferation, migration and invasion of glioma cells. Most importantly, an intracranial glioma xenograft model in nude mice was stablished to observe the influences of silencing PAK1 on tumor growth in vivo. Consistently, we found that knockdown of PAK1 significantly retarded glioma formation. To sum up, these results suggested that PAK1 expression is elevated in glioma patients, inhibition of PAK1 suppresses cell proliferation, migration and invasion of glioma cells and prevents tumor growth in vivo, indicating that PAK1 serves as a novel biomarker and might be a promising target for malignant glioma therapy.

\section{Materials And Methods}

\section{Glioma tissue specimens}

All of the primary human glioma specimens included grade II (12 samples), grade III (12 samples) and grade IV (9 samples) were collected from the Department of Neurosurgery, the Affiliated Hospital of Xuzhou Medical University ranged from September 2016 to September 2019. Besides, no patients underwent antitumor therapies including radiotherapy and chemotherapy before the operation. In addition, 9 cases of normal brain tissue specimens were selected as control. These fresh samples were harvested and immediately frozen in liquid nitrogen. All the glioma samples were histologically diagnosed by three experienced clinical neuropathologists according to the updated WHO Classification of Tumors of the Nervous System in 2016. The histology and clinical data of the glioma samples was shown in Table 1. This study was written informed consent obtained from all patients and approved by the Medical Ethics and Human Clinical Trial Committee of the Affiliated Hospital of Xuzhou Medical University. 


\section{Cell lines and reagents}

The glioma cell lines U87, U251 and human embryonic kidney cell line HEK293T were purchased from the Shanghai Cell Bank, Type Culture Collection Committee, Chinese Academy of Sciences. All cell lines were cultured in Dulbecco's Modified Eagle's medium (DMEM) supplemented with $10 \%$ fetal bovine serum (FBS) (both purchased from Hyclone (Logan, UT, USA)) and supplemented with penicillin $(100 \mathrm{mg} / \mathrm{L}$ ) and streptomycin (100 mg/L, AASIG01-100, Sigma-Aldrich Chemical Company, St Louis, MO, USA) in a condition of $37^{\circ} \mathrm{C}, 5 \% \mathrm{CO}_{2}$ and saturated humidity conditions. Dimethyl sulfoxide (DMSO) were purchased from Sigma-Aldrich (Merck KGaA, Darmstadt, Germany). PolyJet ${ }^{\text {TM }}$ was obtained from SignaGen (Gaithersburg, MD, USA). A potent and selective PAK1 inhibitor IPA-3 was bought from MedChem Express (Monmouth Junction, NJ). A stock solution of IPA-3 was prepared in DMSO, then further diluted with DMEM to yield a final stock concentration of $10 \mu \mathrm{M}$.

\section{Establishment of PAK1 gene silencing model}

According to the complementary DNA (cDNA) sequence of PAK1 gene in GenBank, PAK1 shRNAs were designed by Invitrogen RNAi Designer. Three pairs of specific shRNAs including shPAK1-1, shPAK1-2, shPAK1-3 and a control oligonucleotides were designed as followings:

shPAK1-1 Forward: 5'-

GATCGCTTCAGGCACAGTGTATACTTTCAAGAGAAGTATACACTGTGCCTGAAGCTTTTTTG-3’

shPAK1-1 Reverse: 5'-

AATTCAAAAAAGCTTCAGGCACAGTGTATACTTCTCTTGAAAGTATACACTGTGCCTGAAGC-3'

shPAK1-2 Forward: 5'-

GATCGGGTTGTTATGGAATACTTGGTTCAAGAGACCAAGTATTCCATAACAACCCTTTTTTG-3'

shPAK1-2 Reverse: 5'-

AATTCAAAAAAGGGTTGTTATGGAATACTTGGTCTCTTGAACCAAGTATTCCATAACAACCC-3'

shPAK1-3 Forward: 5'-

GATCGCCTGAACACCTCATTGTATTTTCAAGAGAAATACAATGAGGTGTTCAGGCTTTTTTG-3'

ShPAK1-3 Reverse: 5'-

AATTCAAAAAAGCCTGAACACCTCATTGTATTTCTCTTGAAAATACAATGAGGTGTTCAGGC-3'

Control Forward: 5'-GATCTTCTCCGAACGTGTCACGTTTCAAGAGAACGTGACACGTTCGGAGAATTTTTTG$3^{\prime}$

Control Reverse: 5'-AATTCAAAAAATTCTCCGAACGTGTCACGTTCTCTTGAAACGTGACACGTTCGGAGAA3 '. 
The oligonucleotide $(100 \mu \mathrm{M})$ were annealed and diluted with sterile water 100 times for following use. Subsequently, the annealed oligomers were subcloned into the pLV-shRNA-EGFP(2A)Puro vector by means of both $E c o R I$ and $B a m H I$ cloning sites. The recombinant plasmids were confirmed whether the sequence of shRNA was inserted into the plasmid vector by agarose gel electrophoresis and DNA sequence analysis. The lentiviruses were packaged by three-plasmid-based expression system, namely co-transfecting the core plasmid and the packaging plasmids (psPAX2 and pMD2.G plasmid) in HEK293T cells, which was carried out by PolyJet (SignaGen, Gaithersburg, MD, USA) according to the manufacturer's instructions. The lentiviruses were collected and used for glioma cell infection. $48 \mathrm{~h}$ after infection, the lentivirus-infected cells were cultured in the medium containing $2.5 \mu \mathrm{g} / \mathrm{mL}$ puromycin selection to establish stable shPAK1 cell lines (Sigma, St. Louis, MO, USA).

\section{Cell viability assay}

For the experiment of IPA-3 treatment, $8^{*} 10^{3}$ cells $/ \mathrm{mL}$ U87 and U251 cells were treated with DMSO vehicle and the different concentrations of IPA-3 ranged from 0 to $25 \mu \mathrm{M}$ for $24 \mathrm{~h}$ and then subjected to CCK-8 assay to determine the optimal efficiency concentration of IPA-3 on cell proliferation. We found that U87 and U251 cells displayed more sensitive to $25 \mu \mathrm{M}$ of the half maximal inhibitory concentrations (IC50) value treated with IPA-3 for $24 \mathrm{~h}$. Thus, the IPA-3 at a concentration of $25 \mu \mathrm{M}$ was adopted for the subsequent experiments. For the experiment of PAK1 shRNA treatment, approximately $3 * 10^{3} \mathrm{cell} / \mathrm{s} / \mathrm{mL}$ U87 and U251 cells after infection PAK1 shRNA were plated in 96-well plate per well, each group cells were repeated in four wells, adhered for $20 \mathrm{~h}$ later, then removed the supernatants, at the indicated time $(0,24,48,72,96$ and 120 h), medium mixed with 10\% Cell Counting Kit-8 (CCK-8) reagent (VICMED Life sciences, Xuzhou, China) was added to each well and incubated at $37^{\circ} \mathrm{C}$ for $2 \mathrm{~h}$, the absorbance was measured at $450 \mathrm{~nm}$ by a SynergyMx Multi-Mode Microplate Reader (Biotek, Winooski, VT). All experiments were performed in triplicate independently.

\section{EdU incorporation assay}

This assay aimed to detect cell proliferation using Cell-Light ${ }^{\mathrm{TM}}$ EdU Cell Proliferation Detection Kit (Ruibo Biotech, Guangzhou, China) according to the manufacturer's instructions. For the experiment treated with IPA-3, $8 * 10^{3}$ cells $/ \mathrm{mL}$ U87 and U251 cells were placed in 96 -well plate per well for $20 \mathrm{~h}$, then treated with DMSO vehicle and IPA-3, respectively. Then treated with $50 \mu \mathrm{M}$ 5-ethynyl-20-deoxyuridine (EdU) in $37^{\circ} \mathrm{C}$ for $2 \mathrm{~h}$. Afterwards, the cells were fixed by $4 \%$ paraformaldehydrate for $30 \mathrm{~min}$ and treated with $0.5 \%$ Triton X-100 for $10 \mathrm{~min}$, separately. Thereafter, the cells were incubated with $1 \times$ Apollo $\circledast$ reaction solution for 30 min and stained with $1 \mathrm{X}$ Hoechst33342 reaction cocktail for 30 min in the dark, respectively. Finally, the cells were washed by phosphate buffered saline (PBS) and observed with Olympus IX-71 inverted microscope (Olympus, Japan). The percentage of EdU-positive cells was calculated by the ratio of EdU-positive cells to total Hoechst-stained cells. For the experiment treated with PAK1 shRNA, a total of $8 * 10^{3}$ cells/mL U87 and U251 cells after infection PAK1 shRNA were seeded into 96-well plate, the following disposals were similar to the above descriptions. All experiments were performed in triplicate independently. 


\section{Colony formation assay}

For the experiment of IPA-3 treatment, $1 * 10^{3}$ cells $/ \mathrm{mL}$ U87 and U251 cells after treatment with or without IPA-3 per well were seeded in 6-well plate, each group cells were repeated in three wells, then placed in 37

${ }^{\circ} \mathrm{C}, 5 \% \mathrm{CO}_{2}$ incubator, after 14 days, the plates were washed by PBS, then fixed with $4 \%$ paraformaldehyde for $30 \mathrm{~min}$, finally stained with $0.3 \%$ crystal violet diluted in methanol for $15 \mathrm{~min}$, and visualized immediately on an Olympus IX-71 inverted microscope. Colonies containing more than 50 cells were counted. For the experiment of PAK1 shRNA treatment. A total of $1 * 10^{3}$ cells $/ \mathrm{mL}$ U87 and U251 cells after infection PAK1 shRNA were seeded in 6-well plate, each group cells were repeated in three wells, the following disposals were conducted as previously described. The data was analyzed by the Image $\mathrm{J}$ software (National Institutes of Health, Bethesda, MD). All experiments were performed in triplicate independently.

\section{Cell cycle and cell apoptosis detection}

For the cell cycle experiment, this assay aimed to detect cell cycle distribution using kFlour647 Click-iT EdU Flow Detection Kit (KeyGEN BioTECH, Nanjing, China) according to the manufacturer's instructions.

Cell fixation and permeabilization using the eBioscience ${ }^{\mathrm{TM}}$ Intracellular Fixation \&Permeabilization Buffer Set (Invitrogen). $2 * 10^{5}$ cells $/ \mathrm{mL}$ U87 and U251 cells which were pre-treated with or without IPA-3 were seeded into 6-well plate for $20 \mathrm{~h}$, once attached to the plate, treated with $10 \mu \mathrm{M}$ EdU in $37^{\circ} \mathrm{C}$ for $2 \mathrm{~h}$. Afterwards, cells were harvested and resuspended in $1 \mathrm{ml}$ Fixation Buffer and incubated the at room temperature for 15 minutes in the dark. Afterwards, the cells were resuspended with $1 \times$ Permeabilization Buffer and incubated for 20 min. Next, added Click-iT reaction working mixture to each tube and incubated for 30 minutes at room temperature. Removed the supernatant and resuspended in $1 \mathrm{~mL}$ 1×Permeabilization Buffer containing $5 \mu \mathrm{g} / \mathrm{mL}$ 4,6-diamino-2-phenyl indole (DAPI) (Sigma, St. Louis, MO, USA) to stain the cell nucleus in the dark for $15 \mathrm{~min}$. Finally, the cell suspension was filtered by a $50-\mathrm{mm}$ nylon mesh and immediately analyzed by flow cytometry (BD, Franklin Lakes, NJ, USA). Cell cycle analysis was performed by flowjo 7.6 software. For the experiment of PAK1 shRNA treatment, $2 * 10^{5}$ cells/mL U87 and U251 cells pre-transfected PAK1 shRNA were seeded into 6-well plate, the following disposals was conducted as the above protocol.

For cell apoptosis assay, $2 * 10^{5}$ cells $/ \mathrm{mL}$ U87 and U251 cells pre-treated with or without IPA-3 were performed using PE Annexin V Apoptosis Detection Kit I (BD, Biosciences, USA). The cells were harvested and resuspended in cold PBS, then centrifuged and discarded the supernatants thoroughly. Subsequently, resuspended cells in $1 \times$ Binding Buffer, transfered $100 \mu \mathrm{L}$ of the solution to a $5 \mathrm{ml}$ culture tube, and added $5 \mu \mathrm{L}$ phycoerythrin (PE) Annexin $\mathrm{V}$ and $5 \mu \mathrm{L}$ 7-amino-actinomycin-D (7-AAD), respectively. Vortexed the cells and incubated for $15 \mathrm{~min}$ at room temperature (RT) in the dark. Eventually, added $400 \mu \mathrm{l}$ of $1 \mathrm{X}$ Binding Buffer to each tube, the cell suspensions were filtered by a 50-mm nylon mesh and immediately analyzed by flow cytometry, wherein 10,000 cells were counted from each sample. For the experiment treated with PAK1 shRNA was conducted as previously described. All experiments were performed in triplicate independently. 


\section{Wound healing assay}

The experiment aimed to evaluate the migration behavior of glioma cells. U87 and U251 cells pre-treated with or without IPA-3 were seeded in 6 -well plates. When the cells were at $80 \%$ confluence, the scratches were performed along the straight line with a sterile pipette tip, rinsed with PBS three times to remove detached cells completely. Subsequently, changed serum-free medium and incubated in a condition of 37 ${ }^{\circ} \mathrm{C}, 5 \% \mathrm{CO}_{2}$ and saturated humidity conditions, at the designated time 0 and $24 \mathrm{~h}$, randomly chose six 100x magnification fields per sample around the lesion border by Olympus IX-71 inverted microscope. Finally, counted the migrating cells across the wound to quantify the relative cell migration rates. For the experiment treated with PAK1 shRNA was conducted as previously described. All experiments were performed in triplicate independently.

\section{Transwell migration and invasion assays}

The two experiments were performed using a chemotaxis chambers with polycarbonate nucleopore membrane. The filters with a diameter of $6.5 \mathrm{~mm}$ and pore size of $8 \mu \mathrm{m}$ (Invitrogen). To detect cell invasion ability, firstly, the filters were pre-coated with $50 \mathrm{uL}$ Matrigel $(1 \mathrm{mg} / \mathrm{mL})(B D$, Biosciences, USA), then rehydrated with $100 \mu \mathrm{L}$ FBS-free medium in $37^{\circ} \mathrm{C}$ incubator for $2 \mathrm{~h}$. Subsequently, a density of $6 * 10^{3}$ cells/mL U87 and U251 cells pre-treated with or without IPA-3 suspended with serum-free medium were plated in the upper layer part of each chamber, $600 \mu \mathrm{L}$ culture medium containing $10 \%$ FBS as a source of chemoattractants was added to the each lower chamber and incubated in $37^{\circ} \mathrm{C}$ incubator. $36 \mathrm{~h}$ later, removed the non-invasive cells of the upper chamber with cotton swabs. Afterwards, invasive cells on the lower surface of the filter were fixed with $4 \%$ paraformaldehyde for 30 min and stained with $0.3 \%$ crystal violet ( $w / v$ ) diluted with methanol for 15 min. Finally, five fields of adherent cells in each well were randomly photographed to quantify the cell invasion rates under an Olympus IX71 inverted microscope (Olympus, Japan). The assessment of cell migration ability was the same conduction except that filters were not pre-coated with Matrigel. For the experiment treated with PAK1 shRNA was conducted as previously described. All experiments were performed in triplicate independently.

\section{Western Blotting analysis}

The cells were lysed with RIPA lysis buffer (50 mM Tris/HCl pH 7.4, $150 \mathrm{mM} \mathrm{NaCl}, 1 \mathrm{mM}$ EDTA, 1\% Nonidet P-40, 0.25\% Na-deoxycholate) supplemented with protease and phosphatase inhibitor cocktail, and lysates were incubated on ice for $30 \mathrm{~min}$ to yield a homogenate, subsequently, centrifuged and removed cell debris to obtain the final supernatants. The protein concentrations of whole cell lysates were determined using the BCA protein assay kit (Beyotime, Shanghai, China) according to the manufacturer's instructions. After the protein extract was mixed with 5×SDS buffer (Beyotime, Shanghai, China), the protein was denatured by boiling for $10 \mathrm{~min}$ at $100^{\circ} \mathrm{C}$, then equal amount of protein lysates was subjected to $10-12 \%$ sodium dodecyl sulfate polyacrylamide gel electropheresis (SDS-PAGE) at the voltage of $70 \mathrm{~V}$ to $110 \mathrm{~V}$. And then electrophoretically transferred to polyvinylidene difluoride (PVDF) membrane (Merk Millipore, Billerica, MA, USA) at $110 \mathrm{mV}$, then the membranes were blocked with 3\% 
bovine serum albumin for $60 \mathrm{~min}$ at room temperature, washed with Tris-buffered saline Tween-20 (TBST) $(20 \mathrm{mM}$ Tris pH 7.4, $137 \mathrm{mM} \mathrm{NaCl}$ and $0.1 \%$ Tween-20), subsequently incubated in specific primary antibodies at $4{ }^{\circ} \mathrm{C}$ overnight or at room temperature for $2 \mathrm{~h}$, then the membrane was washed with TBST for three times, followed by incubation with a horseradish peroxidase (HRP)-conjugated secondary antibodies (Cell Signaling Technology (CST), Danvers, MA, USA) at room temperature for $2 \mathrm{~h}$. Using enhanced chemiluminescence (ECL) (Thermo Fisher Scientific, NM, USA) and exposed with a ChemiDocTM Imaging System (Biorad, Hercules, CA, USA). $\beta$-actin was regarded as a loading control. Immunoblots were quantified by densitometry using Image J version 1.46 softwar (National Institute of Health, Bethesda, MD, USA). All experiments were performed in triplicate independently. Antibodies against Bax (50599-2-lg), MMP2 (10373-2-AP), MMP9 (10375-2-AP) and $\beta$-actin (60008-1-lg) were purchased from proteintech (Chicago, USA). Antibodies against PAK1 (\#2602), p21 (\#2647), p53 (\#2524), p38 (\#9212), p-p38 (Thr180/Tyr182, \#9211), CDK4(\#12790), Cyclin D1 (\#2922), Cofilin (D3F9, \#5175) and Cleaved Caspase-3 (\#9664) were purchased from Cell Signaling Technology (Danvers, MA, USA). Antibodies against MDM2 (sc-965) was purchased from Santa Cruz Biotechnology (Santa Cruz, CA, USA). Most antibodies were used in 1:1000 dilutions, unless specified otherwise.

\section{Intracranial mouse model}

All mice experiments were conducted in accordance with protocols approved by the Xuzhou Medical University Animal Care and Use Committee and followed guidelines for animal welfare. For the purpose of establishing intracranial GBM xenografts, 6 to 8-week-old female BALB/c-nude mice were purchased from Beijing Weitong Lihua Experimental Animal Technology Company and housed in Individually Ventilated Caging (IVC) systems in a $12 \mathrm{~h}$ light/dark cycle. A total of $3 * 10^{5} \mathrm{U} 87 \mathrm{cells} / \mathrm{mL}$ expressing mCherry routinely treated by mycoplasma per well were seeded in 6-well plate. When the cell confluence reached $50 \%$, the cells were infected with PAK1 shRNAs and control lentivirus, respectively. After $48 \mathrm{~h}$ of infection, the cells were trypsinized and resuspended with sterile PBS. A total of $5^{\star} 10^{5}$ shPAK 1 and control cells (in $5 \mu \mathrm{L}$ PBS) were collected and injected into the intracranial striatum of nude mice with a stereotactic instrument and a microsyringe. Before injection, mice were anesthetized with $3 \%$ chloral hydrate, subsequently, a hole about $5 \mathrm{~mm}$ in diameter was burred located in $1 \mathrm{~cm}$ anterior and $2 \mathrm{~cm}$ lateral to the bregma, then with the stereotactic guidance, the microsyringe needle penetrated $3 \mathrm{~cm}$ from the calvaria to the striatum and injected cells at a rate of $1 \mu \mathrm{L} / \mathrm{min}[13-16]$. The animals were randomly divided into 3 groups, with 8 mice in each group, including shControl, shPAK1-2, shPAK1-3, respectively. Starting on day $28 \mathrm{~d}$ after tumor cell implantation, sacrificed 2 mice by cervical dislocation from each group every week, and the brain samples were fixed in $4 \%$ paraformaldehyde. Afterwards, performed frozen slices for hematoxylin and eosin (HE) staining to obtain tumor growth status from different groups.

\section{Statistical analysis}

All experiments were performed in triplicate independently. Data were represented as the mean $\pm S D$ as indicated. The Student's t-test (two-tailed) was used for comparison of two groups, comparisons among 
multiple groups were analyzed with One-way analysis of variance (ANOVA). $P<0.05$ was considered statistically significant. All statistical analyses were performed using the SPSS 16.0 software (SPSS Inc., Chicago, IL, USA).

\section{Results}

\section{PAK1 is frequently overexpressed and predicts poor prognosis in glioma patients}

Accumulating evidence has indicated that PAK1 aberrantly amplificates and is also involved in tumorigenesis in breast cancer, ovarian cancer and pancreatic cancer and so on[17-19]. Nevertheless, the study on whether and how PAK1 contributes to carcinogenesis of glioma are still poorly known. In the present study, to investigate the expression of PAK1 in glioma samples, we first examined the differential protein levels in glioma tissues. 33 primary human glioma specimens (12 were grade II, 12 were grade III, and 9 were grade IV) and 9 nontumoral brain tissues from human as control were detected by western blotting. We found that the expressions of PAK1 in gliomas were higher than those in the control.

Furthermore, the protein expression of PAK1 was found to be markedly upregulated in high-grade gliomas (HGG) compared to low-grade gliomas (LGG) patients (Fig. 1a-b). Additionally, to understand the clinical significance of PAK1, an independent cohort of 153 human glioma specimens from The Cancer Genome Atlas (TCGA) database (https://cancergenome.nih.gov/) was used to validate the correlations between PAK1 expressions and survival time of glioma patients. The outcomes provided a provocative evidence that PAK1 expression was positively correlated with glioma grade, and the higher protein level of PAK1, the worse prognosis of glioma patients (Fig. 1C). Taken together, these results provided us the preliminary evidence that PAK1 might be involved in glioma carcinogenesis and progression.

\section{Inhibition of PAK1 suppresses glioma cell proliferation}

Given the high expression of PAK1 in glioma, and PAK1 probably plays an important role in the initiation and progression of glioma. Thus, to explore the role of PAK1 in glioma, we used IPA-3, a potent ATPcompetitive small molecule inhibitor of PAK1, to block the function of PAK1. Firstly, glioma cells U87 and U251 were treated with IPA-3, in a dose and time-dependent manner and subjected to CCK-8 assay. The IPA-3 concentrations ranged from 0 to $25 \mu \mathrm{M}$ for $0,12,24,36,48$ and $60 \mathrm{~h}$. DMSO treated cells were regarded as vehicle control. We found that treatment with IPA-3 retarded the growth of glioma cells both in a dose-dependent (Fig. 2a-2b) and time-dependent manners compared with vehicle control (Fig. 2c-2d), additionally, the efficacy treated with $25 \mu \mathrm{M}$ for $24 \mathrm{~h}$ appeared markedly $(\mathrm{P}<0.05$ for both U87 and U251). Similarly, inhibiting PAK1 through treatment of IPA-3 decreased the ratio of EdU-positive cells in U87 $(P<0.002)$ and U251 $(P<0.004)$ (Fig. 2e-2g). Likewise, colony formation assay exposed to IPA-3 further verified the previous conclusion ( $P<0.01$ for both U87 and U251, Fig. 2h-2j). In addition, we employed PAK1 shRNA to further confirm the prior results. Three shRNA targets including shPAK1-1, shPAK1-2 and shPAK1-3 were subcloned into a lentiviral vector pLV-shRNA-EGFP(2A) Puro and used to produce lentivirus for glioma cell infection. The cell lines were identified by green fluorescent protein (GFP) images and western blotting. As shown in Suppl. Fig. 1, when $48 \mathrm{~h}$ of lentivirus infection, more than $80 \%$ of 
lentivirus-infected U87 and U251 cells exerted GFP fluorescence. The protein level of PAK1 was further confirmed a significant reduction in the shPAK1-2 cells by western blotting (Fig. 2k-2I). Therefore, we chose shPAK1-2 for the subsequent further investigation. In addition, shControl was used regarded as control and shPAK1-3 as well. Obviously, U87 and U251 cells were dramatically decreased after infection of shPAK1-2 compared with transfected shPAK1-3 and shControl $(P=0.005$ and $P=0.008$ for U87 and U251, respectively, Fig. 2m-2n). Besides, The EdU incorporation assay also showed that the proportion of EdU-positive cells infected by shPAK1-2 reduced significantly in U87 ( $P=0.005)$ and U251 ( $P=0.007)$ (Fig. 2o-2r). Furthermore, similar results were also observed in colony formation assay. Compared with the shPAK1-3 and shControl cells, the number of colonies formed in shPAK1-2 cells was significantly reduced $(P<0.001$ for both U87 and U251, Fig. 2s-2u).

Recently, Fang et al. reported that PAK1 promoted acute pancreatitis via p38 and NF-KB pathways[20]. Moreover, Qing et al. found that PAK1-dependent mitogen activated protein kinase (MAPK) pathway activation was required for colorectal cancer cell proliferation[21]. In addition, PAK1 was also involved in regulating expression of relative genes in the DNA damage response pathway[22]. Therefore, the proteins including tumor suppressor proteins p53 and p21 (Cyclin Dependent Kinase Inhibitor-p21) and their downstream target proteins mouse double minute-2 (MDM2) and cyclin-dependent kinase 4 (CDK4) were detected. Giving PAK1 can integrate multiple signal pathways and be involved in the proliferation. Therefore, western blotting was performed to further investigate the mechanisms of how PAK1 affected the proliferation of glioma cell. After infection with shPAK 1 for $72 \mathrm{~h}, \mathrm{U} 87$ and $\mathrm{U} 251$ cells samples were collected and lysed for western blotting. As shown in Fig. 2v-2w, we observed the marked downregulation in p38, p-p38, as well as MDM2 and CDK4 in shPAK1-2 relative to shPAK1-3 and shControl. However, p53 and p21 protein levels were dramatically elevated. In short, treated with inhibitor IPA-3 or shRNA-mediated PAK1 gene silencing suppresses cell proliferation both in U87 and U251 cells.

\section{Inhibition of PAK1 arrests glioma cell cycle progression and facilitates cell apoptosis}

Considering that cell cycle and apoptosis play essential parts in regulating cell growth, and may contribute to proliferation function. Thus, to elucidate whether the PAK1 inhibition-mediated growth restraint was derived from cell cycle arrest and/or apoptosis, firstly, we analyzed the number and distribution of cells in the phases of the cell cycle by flow cytometry. U87 and U251 cells were treated with or without IPA-3 for $24 \mathrm{~h}$ and then subjected to cell cycle assay. The outcome showed that IPA-3 treatment contributed to a rise in the fraction of cells in $\mathrm{G} 1$ phase $(P=0.0042$ and $P=0.036$ for U87 and U251, respectively), however, the number of cells in $S$ phase was less than the group treated with vehicle control ( $P=0.0037$ and $P=0.025$ for U87 and U251, respectively, Fig. 3a-3d). Subsequently, to determine the apoptotic rate of U87 and U251 after treatment with IPA-3, flow cytometry assay was conducted. The data showed that IPA-3 treatment increased the proportions both Annexin $V$ and 7-AAD-positive cell ( $P=0.0046$ and $P=0.0052$ for U87 and U251, respectively, Fig. 3e-3h). After that, the experiment treated with PAK1 shRNA was also conducted. U87 and U251 cells were infected with PAK1 shRNA and control lentivirus, after infection for $48 \mathrm{~h}$, flow cytometry displayed that U87 and U251 cells infected with shPAK12 decreased the percentage of cells in S phase ( $P=0.026$ and $P=0.035$ for U87 and U251, respectively) 
whereas arrested the cells in $\mathrm{G} 1$ phase $(P=0.005$ and $P=0.016$ for U87 and U251, respectively) compared with shPAK1-3 and shControl (Fig. 3i-3k). Similarly, U87 and U251 infected with PAK1 shRNA and subjected to apoptosis assay. As shown in Fig. 3I-3n, U87 and U251 cells of shPAK1-2 enhanced Annexin $V$ percentage and population in 7-AAD-positive cells in comparison to shPAK1-3 and shControl $(P=0.005$ and $P=0.006$ for U87 and U251, respectively). In brief, these findings indicated that inhibition of PAK1 blocks cycle progression and promots apoptosis of glioma cells. Previous studies have indicated that PAK1 participated in Wnt/ $\beta$-catenin signaling pathway, leading to upregulation of Wnt target genes such as cyclin D1[23]. The activated PAK1 in cancer cells brought about a defective mitosis and multipolar spindle orientations. Thus, to explore the underlying mechanism of cell cycle arrest and apoptosis in glioma cells, the expressions of classical markers related to these processes both cell cycle and apoptosis including cyclinD1, B-cell leukemia/lymphoma 2 (Bcl-2), cleaved caspas-3 and Bax (Bcl2 Associated-X Protein) were estimated in response to PAK1 shRNA treatment by western blotting. As shown in Fig. 3o-3p, as expected, the expressions of cyclinD1 and anti-apoptotic protein Bcl-2 in shPAK12 were significantly lower than shPAK1-3 and shControl, in contrast, pro-apoptotic protein cleaved caspas3 , as well as Bax in shPAK1-2 were significantly higher.

Together, these findings above suggested that suppressing PAK1 resultes in S arrest and enhances apoptotic rate of glioma cells through regulating multiple related proteins both cell cycle and apoptosis.

\section{Inhibition of PAK1 suppresses glioma cell migration and invasion}

Due to the high infiltration behaviors of glioma mainly composed of tumor cell migration and invasion. Thus, we next investigate whether inhibition of PAK1 exerts an effect on migration and invasion of glioma cell. Wound healing assay exhibited that the number of cells migrated to the central area at $24 \mathrm{~h}$ was obviously decreased in the group treated with IPA-3 compared to the group of vehicle control ( $P=0.0046$ and $P=0.0057$ for U87 and U251, respectively, Fig. 4a-4d). Moreover, transwell migration assay without matrigel further showed that IPA-3 treament significantly degraded the number of cells migrated to the chamber in U87 $(P<0.001)$ and U251 $(P=0.0005)$ cells (Fig. $4 \mathrm{e}-4 \mathrm{~g})$. Thereafter, the transwell assay containing matrigel was also performed to observe invasion behaviors of U87 and U251 cells treated by IPA-3. The results were consistent with the migration experiment, namely, inhibiting PAK1 notably decreased the number of cells that crossed the matrigel in U87 $(P=0.00061)$ and U251 $(P=0.00075)$ cells (Fig. 4h-4j).

Furthermore, U87 and U251 cells after infection with PAK1 shRNA were also performed by wound healing and transwell assays. Compared with the shPAK1-3 and shControl groups, the number of both migration and invasion in shPAK1-2 group was significantly reduced (Fig. 4k-4r), suggesting that silencing PAK1 markedly repressed glioma cell migration and invasion.

As well known, the epithelial to mesenchymal transition (EMT) exerts an important role in tumor progression. Which has dispalyed in many metastatic cancers such as breast cancer, hepatocellular carcinoma, malignant glioma, and so forth[24]. Indicating that tumor cells probably utilize the process of EMT to obtain their metastatic dissemination. Considering that metalloproteinase 2 (MMP2) and 
metalloproteinase 9 (MMP9) are crucial enzymes for the degradation of the extracellular matrix and may contribute to tumor cell migration and invasion. Therefore, we evaluated the protein levels of MMP2 and MMP9 after infection PAK1 shRNA by western blotting. Correspondingly, one of the representative biomarkers of cell invasion and migration Cofilin was also evaluated. As we expected, silencing PAK1 notably decreased the MMP2 and MMP9 protein levels in U87 and U251 cells. Likewise, Cofilin was well (Fig. 4s-4t). These results suggested that PAK1 is involved in the migration and invasion of glioma cells and knockdown of PAK1 inhibits the migration and invasion of glioma cells.

\section{Knockdown of PAK1 inhibits glioma formation in vivo}

To further clarify whether down-regulation PAK1 also inhibits tumor growth in vivo, we evaluated the effect in situ by establishing xenograft tumor model in nude mice and analyzed the size of gliomabearing mice. The mice were treated with intracranial injection. When the xenograft model was established, starting on $28 \mathrm{~d}$, sacrificed 2 mice from each group every week, then the brain samples were subjected to frozen slices for HE staining to compare tumor growth status of the same section among different groups.

As shown in Fig. 5a and Table 2, no mice loaded tumor in shPAK1-2 group, this was significantly less than those of shPAK1-3 (3 mice) and the shControl (4 mice). Furthermore, the size of xenograft tumor in shPAK1-3 group was obvisouly smaller than that of shControl group under the macroscopic observation. To sum up, the result demonstrated that knockdown of PAK1 tremendously suppresses tumor growth in mice bearing xenograft glioma.

\section{Discussion}

Malignant glioma is the most common type of primary intracranial tumors in adults, and accounts for about 70 percent of human brain tumors[25]. Specially, characterized by its high heterogeneous, widespread consistent infiltration, quick malignant progression. Despite effective treatments including surgical removal, chemotherapy and radiotherapy delay the development and progression to some extent[26-28]. Its current clinical prognosis remains badly poor. Therefore, it is urgent and necessary to explore a novel effective therapeutic target.

Accumulating evidence has indicated that PAK1 performs a crucial part in cytoskeletal dynamics, cell survival, proliferation and EMT of various cancer cells, such as gastric cancer and non-small cell lung cancer[29, 30]. However, its functions in glioma remain unclear. In the present study, we first demonstrated that the protein level of PAK1 was significantly upregulated in various grades of glioma tissues. High expression of PAK1 was closely associated with poor prognosis. Our findings are consistent with the previous report that phosphorylated PAK1 level in the cytoplasm correlates with shorter survival time in patients with glioblastoma[17]. We also proved that inhibition of PAK1 by IPA-3, small molecular inhibitor of PAK1 and PAK1 shRNA suppressed the proliferation, cycle progression, migration, invasion as well as induced apoptosis of glioma cells in vitro. Furthermore, the xenograft inoculated shRNA-mediated PAK1 silencing suppressed the growth of U87 cell in vivo. Totally, these preliminary findings manifested 
that high levels of PAK1 in glioma tissues may accelerate tumor progression. Therefore, PAK1 may be a potential prognostic biomarker for glioma therapy.

As the previous study, PAK1 is believed to be involved in several survival signaling pathways, for instance, activated PAK1 can stimulate PI3K/AKT and NF-KB (nuclear factor KB) pathway, which are important in tumor progression[31,32]. Therefore, in this research, a series of associated protein levels were also detected. We found that knockdown of PAK1 decreased p38 and p-p38 expressions. As well known, p38 is the major subfamily of MAPK cascades and has been widely markedly aberrantly amplified in glioma and involved in the regulation of cell cycle progression and proliferation[33]. It was reported that PAK1dependent MAPK pathway activation was required for colorectal cancer cell proliferation[34]. In short, these results suggested that besides regulating PI3K/AKT and NF-KB pathway, knockdown of PAK1 could also regulate proliferation by inactivating MAPK pathway. Previous study demonstrated that PAK1 gene silencing might decrease proliferation and increase apoptosis of hepatocellular carcinoma cells by regulating the p53/p21 pathway[35]. In this study, we showed that knockdown of PAK1 increased the expressions of p53 and p21, and decreased the expressions of CyclinD1, MDM2 and CDK4. Guardian of the genome p53 is a tumour suppressor that regulates the expression of multifarious genes involved in apoptosis, growth arrest, inhibition of cell cycle progression and accelerated DNA repair in response to genotoxic or cellular stress[36]. Nonphosphorylated p53 is ubiquitylated degradation by the MDM2, which acts as a ubiquitin ligase and major regulator of p53. p53 is involved in cell-cycle inhibition by stimulating the expression of $p 21[37]$. Additionally, recent studies revealed that PAK1 overexpression promotes cell proliferation in cutaneous T cell lymphoma via suppression of p21[38]. p21 is an inhibitor of CDKs (Cyclin-Dependent Kinases) that regulates the cell cycle via perturbation of its partner cyclins. cyclins associate with CDKs to regulate their activity and the progression of the cell cycle through specific checkpoints. Disruption of Cyclin action leads to either cell cycle arrest, or to uncontrolled cell cycle proliferation[39]. p53 stimulates two major apoptotic pathways: extrinsic pathways and intrinsic pathways, resulting in a cascade of activation of caspases and high expression of the Bcl-2 family proteins, respectively, which in turn induce apoptosis[40]. Our findings demonstrated that knockdown of PAK1 increased the expressions of pro-apoptotic proteins Cleaved caspase- 3 and Bax, while decreased the expression of the anti-apoptotic protein Bcl-2.

An increasing number of studies have shown that the EMT is considered as a crucial mechanism which tumor cells utilize this process to acquire migration and invasion properties in metastastic cancers[24]. It is reported that Cofilin induces the epithelial-mesenchymal transition of gastric cancer cells by promoting cytoskeletal rearrangement. Cofilin is an actin-binding protein by promoting cell migration and invasion, facilitating tumor metastasis[41]. Besides, the recent study also find that CXCL10 accelerates EMT and metastasis by MMP-2 in hepatocellular carcinoma[42]. Matrix metalloproteinases (MMPs) have been regarded as proteolytic enzymes which play an important role in tumor invasion and migration through degrading basement membrane and extracellular matrix (ECM) components[43]. In the study, we found that knockdown of PAK1 decreased the expressions of Cofilin, MMP2 and MMP9. Thus, we speculate that silencing PAK1 inhibited EMT programs may be via targeting decreasing MMP2, MMP9 and Cofilin. 


\section{Conclusion}

In conclusion, our study elucidated that PAK1 significantly overexpressed in various grades of glioma tissues. High expression of PAK1 was closely associated with poor prognosis. Furthermore, PAK1 inhibition restrained the proliferation, cycle progression, migration, invasion as well as induced apoptosis of glioma cells. However, the whole underlying mechanism is still unclear. Therefore, the potential mechanism of how PAK1 promotes glioma development needs to further explore. Targeting PAK1 may be a novel therapeutic strategy for malignant glioma.

\section{Declarations}

\section{Supplementary information}

Additional file 1: Figure S1. Cell morphology, green fluorescent with three shRNAs after infection for $48 \mathrm{~h}$. BF, bright field. GFP, fluorescence. Scale bar, $100 \mu \mathrm{m}$.

\section{Acknowledgements}

Not applicable.

\section{Authors' contributions}

QY was responsible for experimental design and data collection, TC was responsible for article writing and figures editing, KZ and QM were responsible for clinical data collection and performing clinical validation. ZS, LW and RW were responsible for proofreading. HS was responsible for the review. All authors read and approved the final manuscript.

\section{Funding}

This work was supported by the National Natural Science Foundation of China (81672490 and 81874081), the Jiangsu Provincial Medical Youth Talent (QNRC2016784) and the Qinglan Project of Jiangsu Province.

\section{Availability of data and materials}

Not applicable.

\section{Ethics approval and consent to participate}

The study was approved by the Ethics Committee of the Xuzhou Medical University and samples were obtained with written informed consent from all patients, and all informed consent were conducted in accordance with the Declaration of Helsinki.

\section{Consent for publication}


Informed consent was obtained from all patients.

\section{Competing interests}

The authors declare that they have no competing interests.

\section{Author details}

${ }^{a}$ Institute of Nervous System Diseases, Xuzhou Medical University, Xuzhou, Jiangsu, China

${ }^{b}$ Central Laboratory, the Affiliated Hospital of Xuzhou Medical University, Xuzhou, Jiangsu, China

${ }^{c}$ Institute of Digestive Diseases, Xuzhou Medical University, Xuzhou, Jiangsu, China

\section{References}

1. Han, X., H. Li, Y. Zhang, J. Qin, Q. Yang, et al., Brain lipid-binding protein promotes proliferation and modulates cell cycle in C6 rat glioma cells. International Journal of Oncology, 2017. 51(5): p. 14391448.

2. Chen, R., M. Smith-Cohn, A.L. Cohen, and H. Colman, Glioma Subclassifications and Their Clinical Significance. Neurotherapeutics, 2017. 14(2): p. 1-14.

3. Liang, X., Z. Dong, W. Bin, N. Dekang, Z. Xuhang, et al., PAX3 Promotes Proliferation of Human Glioma Cells by WNT/B-Catenin Signaling Pathways. Journal of Molecular Neuroscience, 2019. 68(1): p. 66-77.

4. Dušan, M., F. Elke, G.A. Ligia, and N. Gabriele, Increased radiosensitivity and radiothermosensitivity of human pancreatic MIA PaCa-2 and U251 glioblastoma cell lines treated with the novel Hsp90 inhibitor NVP-HSP990. Radiation Oncology, 2013. 8(1): p. 42-42.

5. Feng, J., P.F. Yan, H.Y. Zhao, F.C. Zhang, W.H. Zhao, et al., SIRT6 suppresses glioma cell growth via induction of apoptosis, inhibition of oxidative stress and suppression of JAK2/STAT3 signaling pathway activation. Oncology Reports, 2016. 35(3): p. 1395.

6. Janbazian, L., J. Karamchandani, and S. Das, Mouse models of glioblastoma: lessons learned and questions to be answered. Journal of Neuro-Oncology, 2014. 118(1): p. 1-8.

7. Kumar, R. and D.Q. Li, Chapter Four - PAKs in Human Cancer Progression : From Inception to Cancer Therapeutic to Future Oncobiology. Advances in Cancer Research, 2016. 130: p. 137.

8. Rane, C.K. and A. Minden, P21 activated kinase signaling in cancer. Seminars in Cancer Biology, 2018.

9. Rane, C. and A. Minden, P21 activated kinases: structure, regulation, and functions. Small Gtpases, 2014. 5(1).

10. Maria, R., S. Galina, K. Rachelle, and C. Jonathan, PAK signalling during the development and progression of cancer. Nature Reviews Cancer, 2014. 14(1): p. 13-25. 
11. Delorme-Walker, V.D., J.R. Peterson, C. Jonathan, C.M. Waterman, D. Gaudenz, et al., Pak1 regulates focal adhesion strength, myosin IIA distribution, and actin dynamics to optimize cell migration. Journal of Cell Biology, 2011. 193(7): p. 1289-1303.

12. Ong, C.C., A.M. Jubb, P.M. Haverty, Z. Wei, T. Victoria, et al., Targeting p21-activated kinase 1 (PAK1) to induce apoptosis of tumor cells. Proc Natl Acad Sci U S A, 2011. 108(17): p. 7177-7182.

13. Huang, K., C. Fang, K. Yi, X. Liu, H. Qi, et al., The role of PTRF/Cavin1 as a biomarker in both glioma and serum exosomes. Theranostics, 2018. 8(6): p. 1540-1557.

14. Zhang, Z., Z. Wang, K. Huang, Y. Liu, C. Wei, et al., PLK4 is a determinant of temozolomide sensitivity through phosphorylation of IKBKE in glioblastoma. Cancer Letters, 2019. 443: p. 91-107.

15. Zheng, Q., L. Han, Y. Dong, J. Tian, W. Huang, et al., JAK2/STAT3 targeted therapy suppresses tumor invasion via disruption of the EGFRVIII/JAK2/STAT3 axis and associated focal adhesion in EGFRvIIIexpressing glioblastoma. Neuro-oncology, 2014. 16(9): p. 1229.

16. Chen, L., L. Han, K. Zhang, Z. Shi, J. Zhang, et al., VHL regulates the effects of miR-23b on glioma survival and invasion via suppression of HIF-1a/VEGF and $\beta$-catenin/Tcf-4 signaling. Neurooncology, 2012. 14(8): p. 1026.

17. Hiroshi, A., Y. Tomohisa, F. Keishi, A.M. Tari, S. Raymond, et al., Phosphorylated Pak1 level in the cytoplasm correlates with shorter survival time in patients with glioblastoma. Clinical Cancer Research, 2007. 13(1): p. 6603-6609.

18. Yang, Z., H. Wang, L. Xia, L. Oyang, Y. Zhou, et al., Overexpression of PAK1 Correlates with Aberrant Expression of EMT Markers and Poor Prognosis in Non-Small Cell Lung Cancer. Journal of Cancer, 2017. 8(8): p. 1484-1491.

19. Al-Azayzih, A., F. Gao, and P.R. Somanath, P21 activated kinase-1 mediates transforming growth factor $\beta 1$-induced prostate cancer cell epithelial to mesenchymal transition. BBA - Molecular Cell Research, 2015. 1853(5): p. 1229-1239.

20. Zhu, M., Y. Xu, W. Zhang, T. Gu, and D. Wang, Inhibition of PAK1 alleviates cerulein-induced acute pancreatitis via p38 and NF-KB pathways. Bioscience Reports, 2019. 39(3).

21. Qing, H., W. Gong, Y. Che, X. Wang, L. Peng, et al., PAK1-dependent MAPK pathway activation is required for colorectal cancer cell proliferation. Cancer Science, 2012. 33(4): p. 985-994.

22. Prudnikova, T.Y., O. Villamarcruz, S.J. Rawat, K.Q. Cai, and J. Chernoff, Effects of p21-activated kinase 1 inhibition on 11q13-amplified ovarian cancer cells. Oncogene, 2016. 35(17): p. 2178-2185.

23. Hans, C., Wnt/beta-catenin signaling in development and disease. Cell, 2006. 127(3): p. 469-480.

24. Brabletz, T., R. Kalluri, M.A. Nieto, and R.A. Weinberg, EMT in cancer. Nature Reviews Cancer, 2018. 18(2): p. 128-134.

25. Zhu, Y., X. Zhang, L. Wang, Z. Ji, M. Xie, et al., Loss of SH3GL2 promotes the migration and invasion behaviours of glioblastoma cells through activating the STAT3/MMP2 signalling. Journal of Cellular \& Molecular Medicine, 2017. 21(11): p. 2685. 
26. Zhu, H., D. Chen, J. Tang, C. Huang, S. Lv, et al., Overexpression of centrosomal protein 55 regulates the proliferation of glioma cell and mediates proliferation promoted by EGFRvIll in glioblastoma U251 cells. Oncology Letters, 2018. 15(2): p. 2700.

27. Zhang, T., D. Ji, P. Wang, D. Liang, L. Jin, et al., The atypical protein kinase RIOK3 contributes to glioma cell proliferation/survival, migration/invasion and the AKT/mTOR signaling pathway. Cancer Letters, 2017. 415: p. 151.

28. Choi, P.J., R.S. Tubbs, and R.J. Oskouian, Emerging Cellular Therapies for Glioblastoma Multiforme. Cureus, 2018. 10(3): p. e2305.

29. Yeo, D., H. He, G. Baldwin, and M. Nikfarjam, P-038 * FRAX597, a PAK1 inhibitor, synergises with gemcitabine in the reduction of pancreatic cancer growth. Annals of Oncology.

30. Park, J., J.M. Kim, J.K. Park, S. Huang, S.Y. Kwak, et al., Association of p21-activated kinase-1 activity with aggressive tumor behavior and poor prognosis of head and neck cancer. Head \& Neck, 2015. 37(7): p. 953-963.

31. Romero, L.E.A., O.V. Cruz, and J. Chernoff, Abstract 4865: Pak1 links the Wnt/ $\beta$-catenin pathway to ErbB2 signaling in breast cancer cells. Cancer Research, 2012. 72(8 Supplement): p. 4865-4865.

32. Wu, D.W., T.C. Wu, C.Y. Chen, and H. Lee, PAK1 Is a Novel Therapeutic Target in Tyrosine Kinase Inhibitor-Resistant Lung Adenocarcinoma Activated by the PI3K/AKT Signaling Regardless of EGFR Mutation. Clinical Cancer Research, 2017. 22(21): p. 5370-5382.

33. Huang, B.M., P 046 - Cordycepin induced MA-10 mouse Leydig tumor cell apoptosis by regulating p38 MAPKs and PI3K/AKT signaling pathways. Free Radical Biology \& Medicine, 2017. 108: p. S32.

34. Qing, H., W. Gong, Y. Che, X. Wang, L. Peng, et al., PAK1-dependent MAPK pathway activation is required for colorectal cancer cell proliferation. Tumor Biology.

35. Zhang, Z.L., G. Liu, L. Peng, C. Zhang, Y.M. Jia, et al., Effect of PAK1 gene silencing on proliferation and apoptosis in hepatocellular carcinoma cell lines MHCC97-H and HepG2 and cells in xenograft tumor. Gene Therapy, 2018.

36. Bykov, V., S.E. Eriksson, J. Bianchi, and K.G. Wiman, Targeting mutant p53 for efficient cancer therapy. Nature Reviews Cancer, 2018. 18(2): p. 89.

37. Wang, S., Y. Zhao, A. Aguilar, D. Bernard, and C.Y. Yang, Targeting the MDM2-p53 Protein-Protein Interaction for New Cancer Therapy: Progress and Challenges. Cold Spring Harb Perspect Med, 2017. 7(5): p. a026245.

38. Wang, Y., X. Gu, W. Li, Q. Zhang, and C. Zhang, PAK1 overexpression promotes cell proliferation in cutaneous $T$ cell lymphoma via suppression of PUMA and p21. Journal of Dermatological Science, 2018. 90(1): p. S0923181118300021.

39. Brooks, J.K., N.G. Nikitakis, B.F. Frankel, J.C. Papadimitriou, and J.J. Sauk, Oral inflammatory myofibroblastic tumor demonstrating ALK, p53, MDM2, CDK4, pRb, and Ki-67 immunoreactivity in an elderly patient. Oral Surgery Oral Medicine Oral Pathology Oral Radiology \& Endodontics, 2005. 99(6): p. 716-726. 
40. Xie, X., O.P. Clausen, A.P. De, and M. Boysen, The prognostic value of spontaneous apoptosis, Bax, Bcl-2, and p53 in oral squamous cell carcinoma of the tongue. Cancer, 2015. 86(6): p. 913-920.

41. Wang, H., L. Tao, J. Feng, G. Hao, X. Dai, et al., Cofilin 1 induces the epithelial-mesenchymal transition of gastric cancer cells by promoting cytoskeletal rearrangement. Oncotarget, 2017. 8(24).

42. Ren, T., L. Zhu, and M. Cheng, CXCL 10 accelerates EMT and metastasis by MMP-2 in hepatocellular carcinoma. American Journal of Translational Research, 2017. 9(6): p. 2824-2837.

43. Wang, J., H. Hirose, G. Du, K. Chong, E. Kiyohara, et al., P-REX1 amplification promotes progression of cutaneous melanoma via the PAK1/P38/MMP-2 pathway. Cancer Letters, 2017. 407: p. 66.

\section{Tables}

Table 1 Clinical data of glioma patient 


\begin{tabular}{|c|c|c|c|c|c|}
\hline Case ID & Grade & Pathology & Age & Gender & Location \\
\hline 1014888 & II & Astrocytoma & 41 & Female & Right-Frontal lobe \\
\hline 1305927 & II & Astrocytoma & 59 & Female & Right-Frontal lobe \\
\hline 1161905 & II & Oligoastrocytoma & 47 & Male & Left-Temporal lobe \\
\hline 1376626 & II & Astrocytoma & 54 & Male & Right-Frontal and Parietal lobe \\
\hline 1356449 & II & Astrocytoma & 37 & Male & Right-Frontal lobe \\
\hline 1190502 & II & Astrocytoma & 44 & Male & Left-Frontal lobe \\
\hline 1196273 & II & Astrocytoma & 45 & Female & Left-Temporal lobe \\
\hline 1221743 & II & Astrocytoma & 50 & Female & Right-Temporal lobe \\
\hline 1327564 & II & Oligoastrocytoma & 41 & Male & Right-Frontal lobe \\
\hline 1236896 & II & Oligoastrocytoma & 55 & Male & Right-Frontal and Parietal lobe \\
\hline 1197520 & II & Oligoastrocytoma & 44 & Male & Right-Temporal lobe \\
\hline 1234728 & II & Astrocytoma & 48 & Male & Right-Temporal lobe \\
\hline 1260826 & III & Anaplastic-Astrocytoma & 46 & Female & Left-Occipital lobe \\
\hline 1116337 & III & Anaplastic- Oligodendroglioma & 47 & Male & Left-Parietal lobe \\
\hline 1258033 & III & Anaplastic- Oligodendroglioma & 54 & Female & Right-Temporal lobe \\
\hline 1259365 & III & Anaplastic-Astrocytoma & 49 & Female & Right-Temporal lobe \\
\hline 1191197 & III & Anaplastic-Astrocytoma & 60 & Female & Right-Frontal and Parietal lobe \\
\hline 1257321 & III & Anaplastic-Astrocytoma & 43 & Female & Left-Frontal and Parietal lobe \\
\hline 1237351 & III & Astrocytoma \& Anaplastic-Astrocytoma & 55 & Male & Right-Frontal lobe \\
\hline 1246343 & III & Astrocytoma \& Anaplastic-Astrocytoma & 52 & Male & Right-Frontal and Temporal lobe \\
\hline 1190502 & III & Anaplastic- Oligodendroglioma & 66 & Male & Left-Frontal lobe \\
\hline 1182956 & III & Anaplastic- Oligodendroglioma & 68 & Male & Right-Parietal lobe \\
\hline 1164493 & III & Astrocytoma \& Anaplastic-Astrocytoma & 56 & Male & Right-Temporal lobe \\
\hline 1164248 & III & Anaplastic- Oligodendroglioma & 55 & Female & Left-Frontal lobe \\
\hline 1110886 & IV & Glioblastoma & 37 & Female & Right-Temporal lobe \\
\hline 1305743 & IV & Glioblastoma & 47 & Female & Right-Frontal lobe \\
\hline 1204425 & IV & Glioblastoma & 41 & Male & Right-Parietal and Occipital lobe \\
\hline 1214169 & IV & Glioblastoma & 49 & Male & Right-Frontal lobe \\
\hline 1302359 & IV & Glioblastoma & 60 & Female & Left-Temporal lobe \\
\hline 1373335 & IV & Glioblastoma & 61 & Female & Right-Temporal lobe \\
\hline 1374495 & IV & Glioblastoma & 35 & Female & Right-Frontal lobe \\
\hline 1156841 & IV & Glioblastoma & 64 & Male & Right-Frontal and Parietal lobe \\
\hline 1145683 & IV & Glioblastoma & 47 & Male & Left-Temporal lobe \\
\hline
\end{tabular}

Table 2 The statistics of mice bearing tumor

\begin{tabular}{ll}
\hline group & Number of mice bearing tumor \\
\hline shControl & $4(8)$ \\
shPAK1-2 & $0(8)$ \\
shPAK1-3 & $3(8)$ \\
\hline
\end{tabular}


Table 2. The statistics of mice bearing tumor and total mice among three groups: shControl, shPAK1-2 and PAK13.

\section{Figures}

A

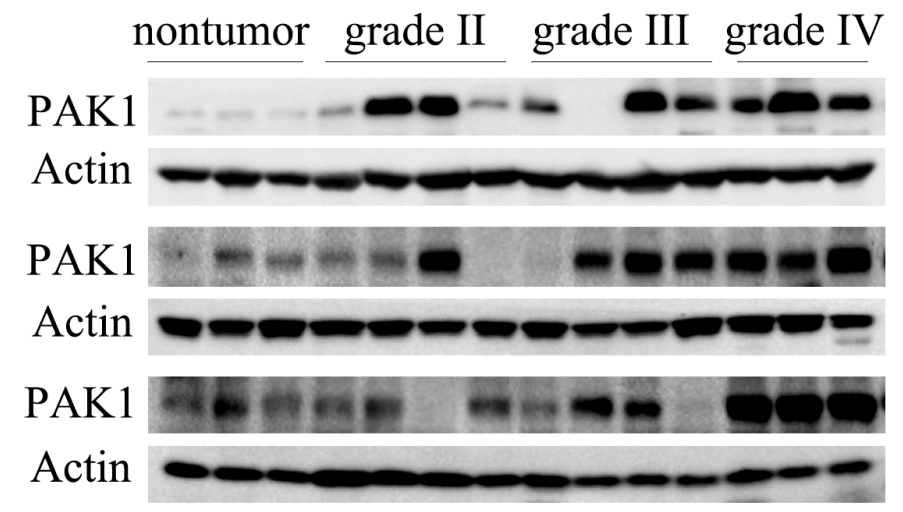

$\mathrm{B}$

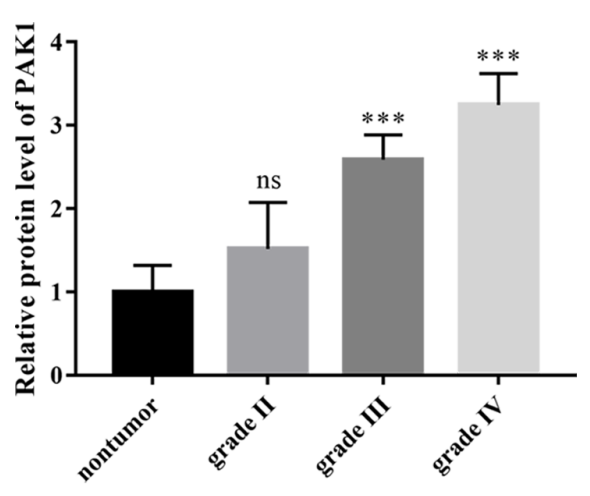

C

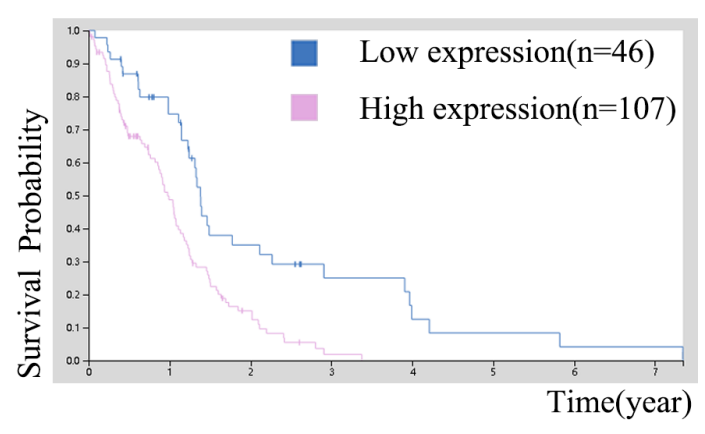

\section{Figure 1}

PAK1 is frequently overexpressed and predicts poor prognosis in glioma patients a-b. Western blotting analysis and quantitation of PAK1 expressions in 33 primary human glioma tissues (12 were grade II, 12 were grade III, and 9 were grade IV) and 9 normal brain tissues. Relative density of immunoblot was normalized to the normal. Actin regarded as loading control. PAK1, p21-activated kinase 1. c. KaplanMeier analysis of overall survival curves of gliomas patient from The Cancer Genome Atlas (TCGA) database analysis. ${ }^{*} P<0.05,{ }^{*} P<0.01$ and ${ }^{* * *} P<0.001$. 


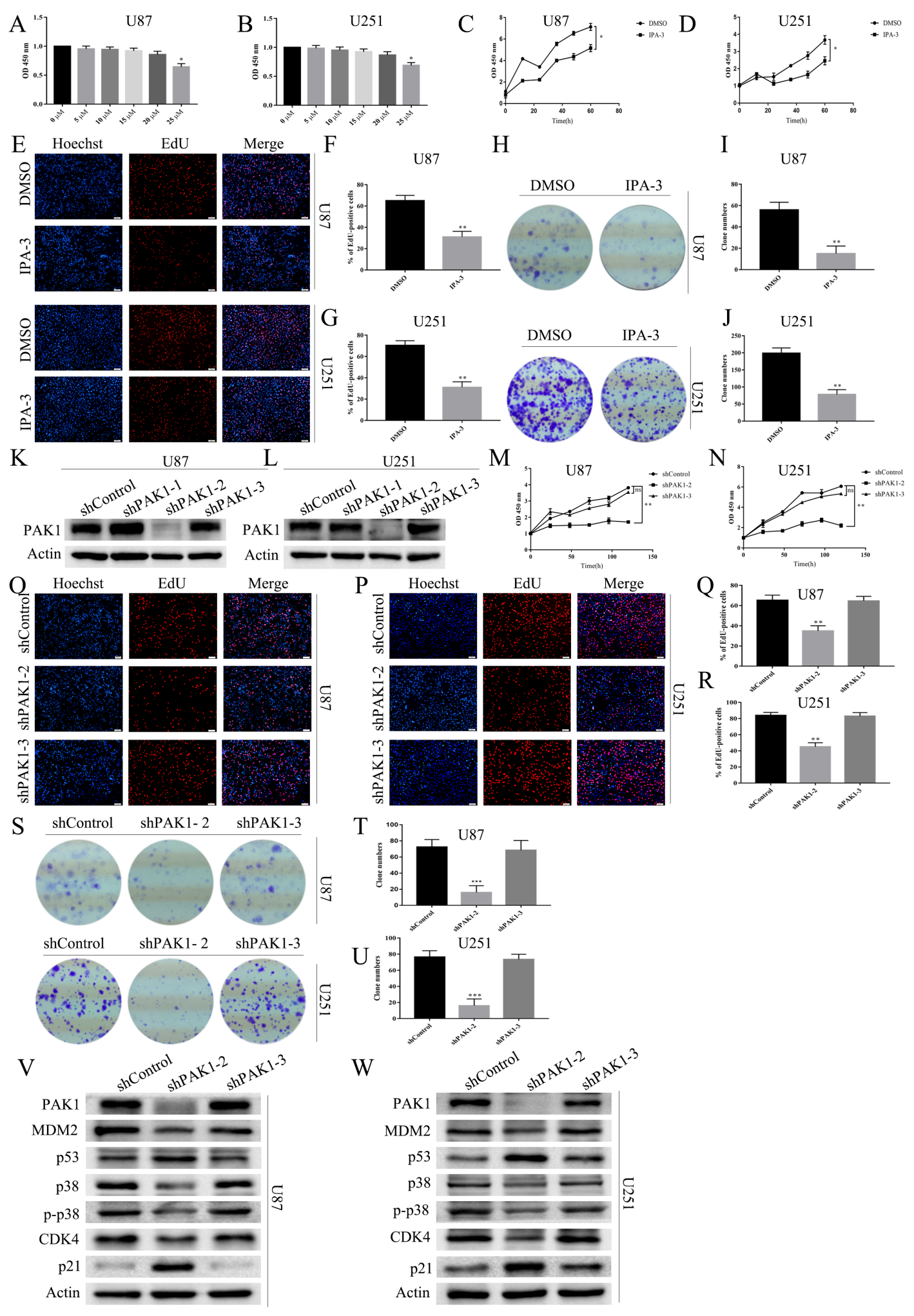

Figure 2

Inhibition of PAK1 suppresses proliferation of glioma cells a-d. CCK-8 assay intended to detect the optimal inhibitory concentration $(0,5,10,15,20,25 \mu \mathrm{M})$ and time $(0,12,24,36,48,60 \mathrm{~h})$ of IPA-3 on U87 and U251 cells. IPA-3, p21-activated kinase inhibitor III. OD, optical density, $P<0.05$. e-j. EdU and colony formation assays aimed to evaluate the U87 and U251 cells proliferation after treatment with small molecule inhibitor IPA-3 $(25 \mu \mathrm{M})$, respectively. Representative images displayed the Hoechst-stained 
nuclei (blue) and EdU-positive cells (red). scale bar, $100 \mu \mathrm{m}$. k-I. The silencing efficiency of PAK1 at protein level with three shRNAs after infection for $48 \mathrm{~h}$. Actin regarded as loading control. m-u. CCK-8, EdU and colony formation assays were used to evaluate the U87 and U251 cells proliferation after inhibition by PAK1 shRNA, respectively. v-w. Expressions of MDM2, p53, p38, p-p38, CDK4 and p21 in U87 and U251 cells after infection shPAK1. Actin regarded as loading control. All experiments were performed in triplicate independently. PAK1, p21-activated kinase 1. ${ }^{*} P<0.05$, ${ }^{*} \mathrm{P}<0.01$ and ${ }^{*} * * \mathrm{P}<0.001$.
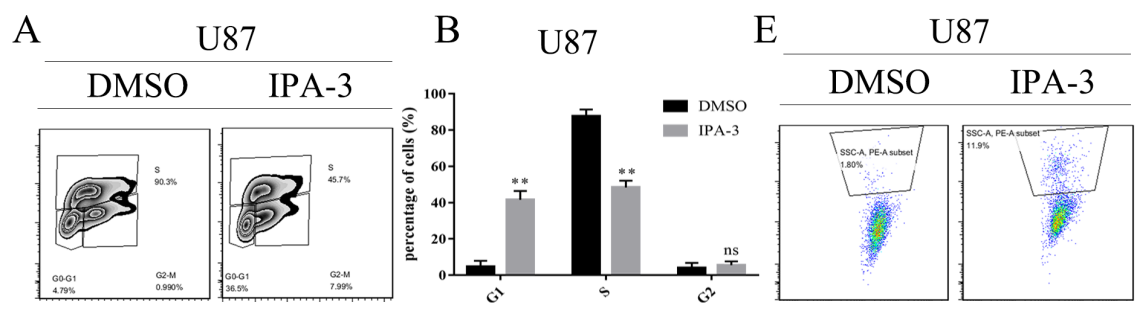

F U87

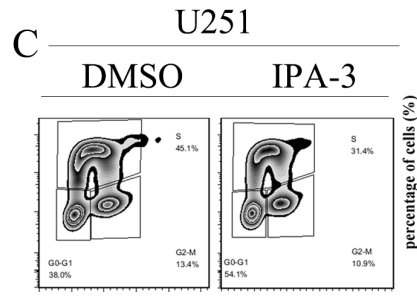

D U251
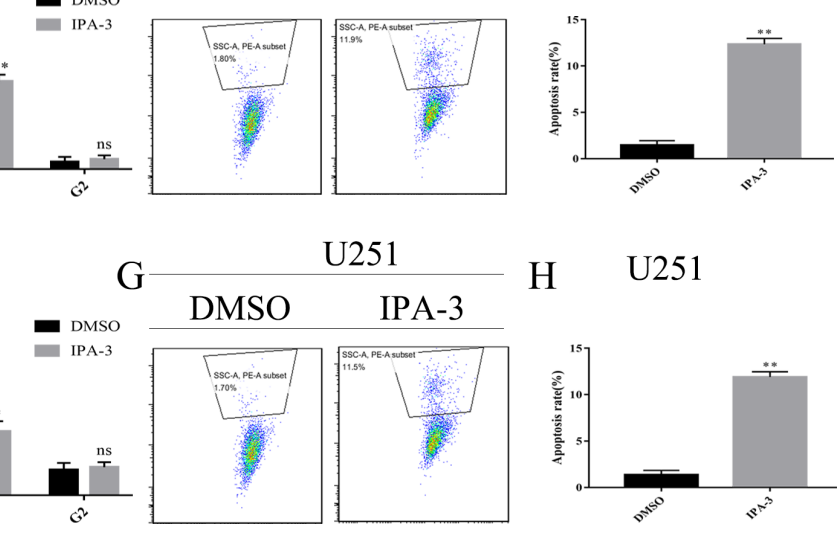

$\mathrm{H} \quad \mathrm{U} 251$

I shControl shPAK1-2 shPAK1-3
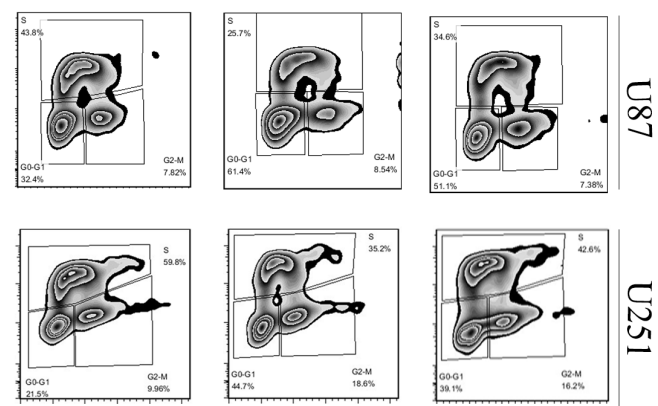

$\mathrm{J}$

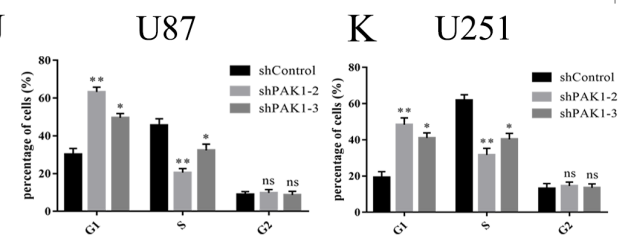

$\mathrm{O}$
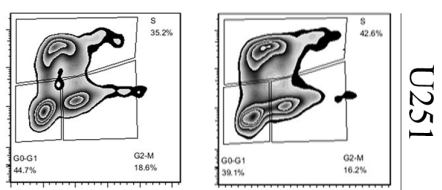

$\mathrm{U} 25$

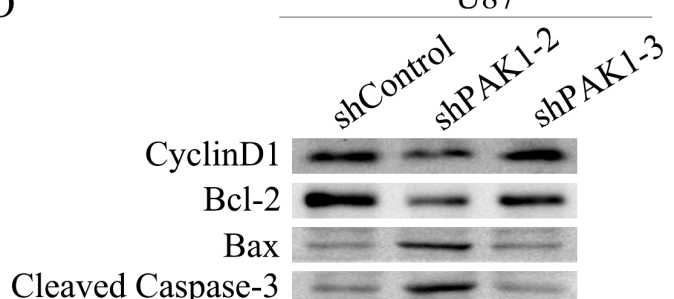

PAK1 Actin

Cleaved Casp

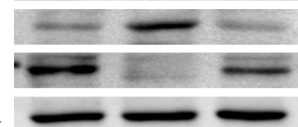

L shControl shPAK1-2 shPAK1-3
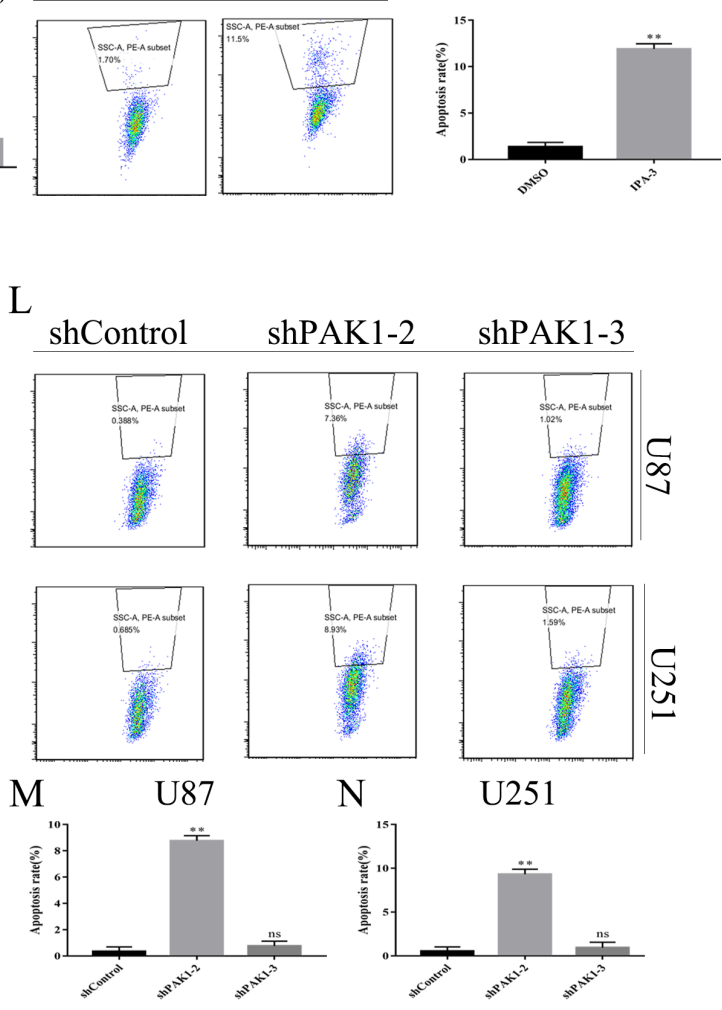

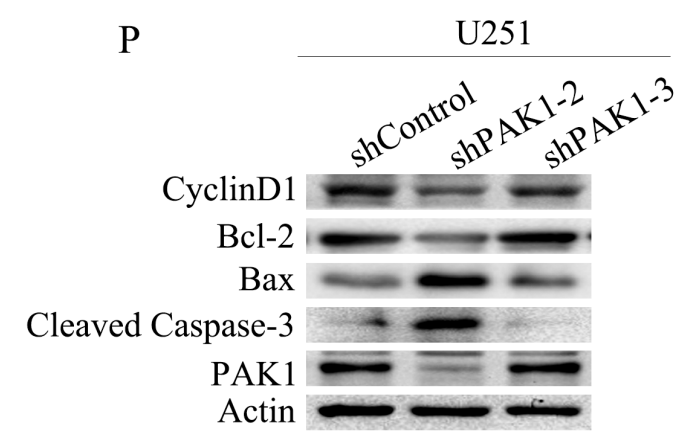

Figure 3 
Inhibition of PAK1 arrests glioma cell cycle progression and facilitates cell apoptosis a-h. Flow cytometry aimed to evaluate cell cycle (3a-3d) and apoptotic rate (3e-3h) of U87 and U251 after treatment with IPA-3 $(25 \mu \mathrm{M})$, respectively. The percentage of cells at different phases was exhibited. IPA-3, p21-activated kinase inhibitor III. i-n. Flow cytometry aimed to examine cell cycle (3i-3k) and apoptotic rate (3l-3n) of U87 and U251 after treatment with PKA1 shRNA, respectively. The percentage of apoptosis cells was exhibited. o-p. Western blotting analysis showed knockdown of PAK1 on expression of classical markers both cell cycle and apoptosis including CyclinD1, Bcl-2, Bax and cleaved caspas-3. Actin regarded as loading control. All experimental data were carried out in triplicate independently. PAK1, p21-activated kinase $1 .{ }^{*} P<0.05,{ }^{\star *} \mathrm{P}<0.01$ and ${ }^{\star *} * \mathrm{P}<0.001$. 

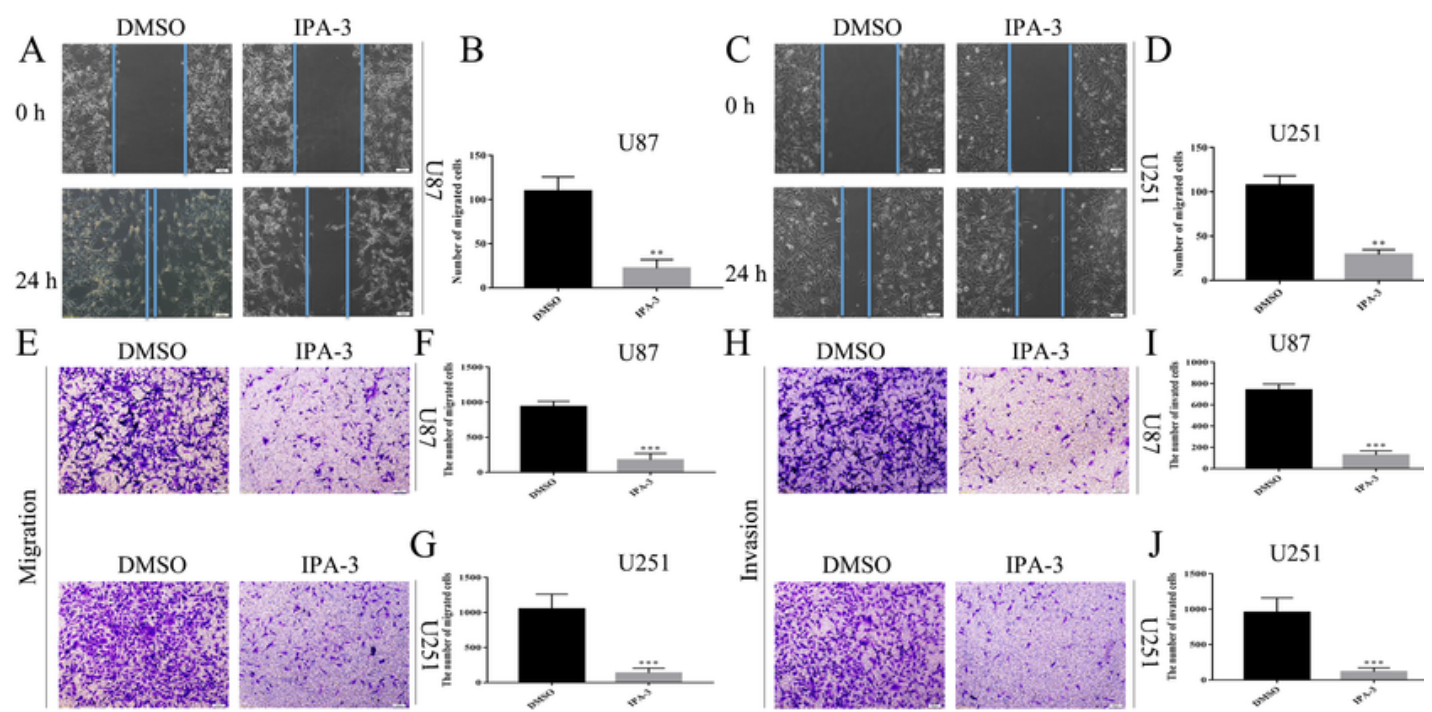

IPA-3
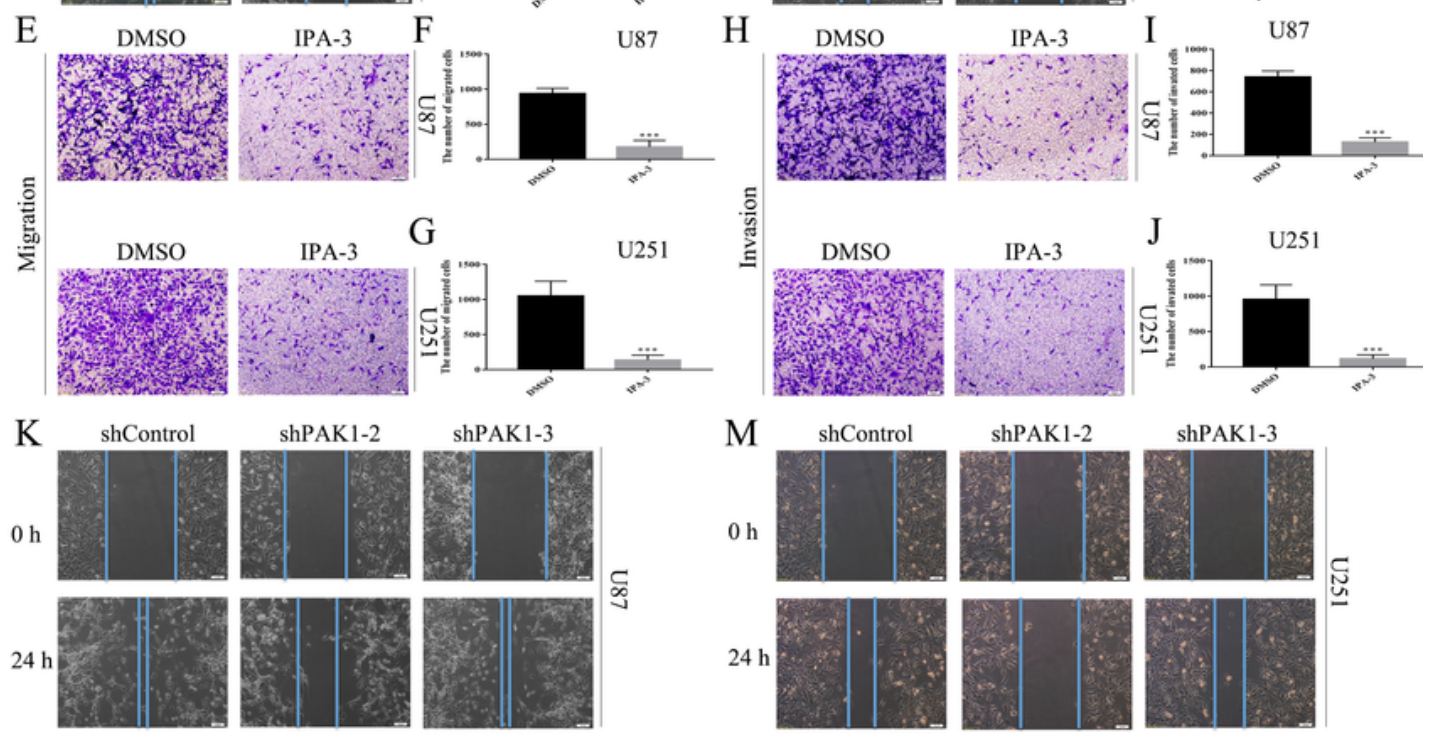

shPAK1-3
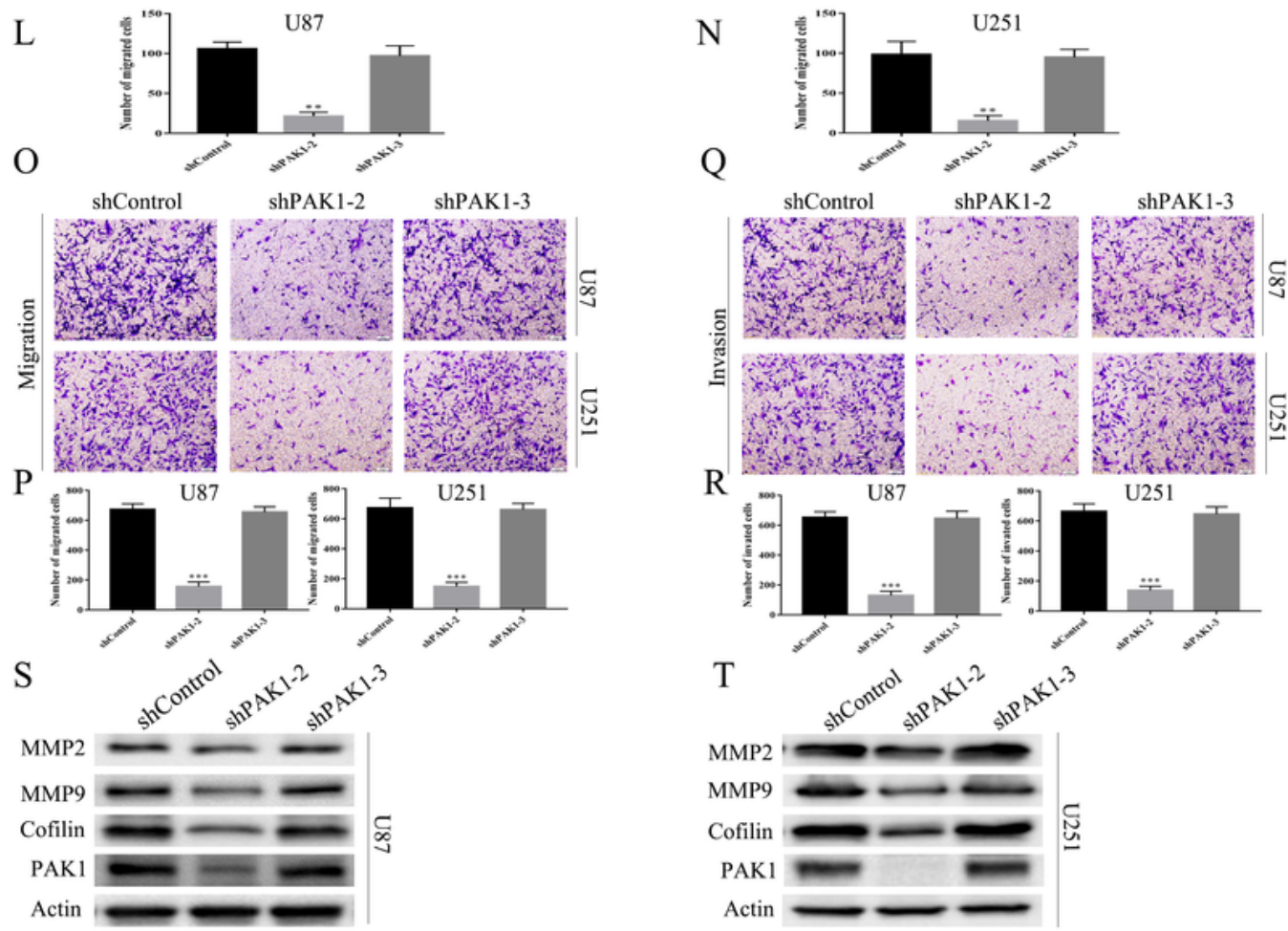

\section{Figure 4}

Inhibition of PAK1 suppresses glioma cell migration and invasion a-j. The wound healing and transwell assays were performed to evaluate the U87 and U251 cells migration (Fig. 4a-4g) and invasion (Fig. 4h4j) after treatment by inhibitor IPA-3 $(25 \mu \mathrm{M})$. IPA-3, p21-activated kinase inhibitor III. k-r. The wound healing and transwell assays aimed to evaluate the U87 and U251 cells migration (Fig. 4k-4p) and invasion (Fig. 4q-4r) after infection by PKA1 shRNA. Representative images and quantification of U87 
and U251 cell migration and invasion were showed. scale bar, $100 \mu \mathrm{m}$. s-t. Western blotting analysis showed knockdown of PAK1 on expression of invasion and migration associated biomarkers including MMP2, MMP9 and Cofilin. Actin regarded as loading control. All experimental data were carried out in triplicate. ${ }^{*} \mathrm{P}<0.05,{ }^{*} \mathrm{P}<0.01$ and ${ }^{* * *} \mathrm{P}<0.001$.

A

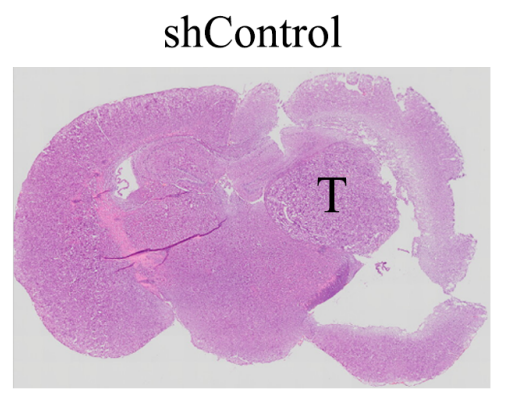

shPAK1-2

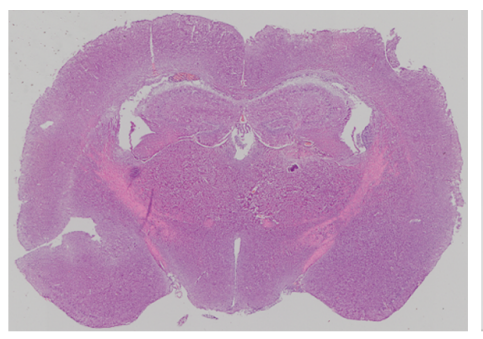

shPAK1-3

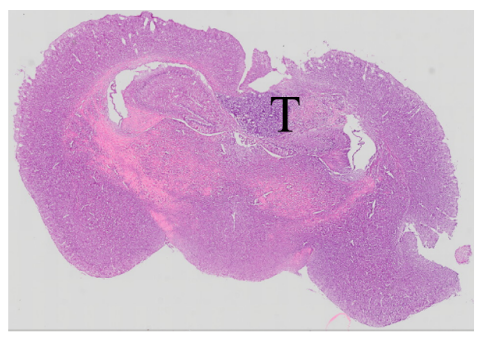

\section{Figure 5}

Knockdown of PAK1 inhibits glioma formation in vivo a. Representative HE staining images of mice brain bearing tumor. Scale bar, $100 \mu \mathrm{m}$. T, tumor.

\section{Supplementary Files}

This is a list of supplementary files associated with this preprint. Click to download.

- Additionalfile1.docx 